\title{
CARACTERIZACIÓN FITOLÍTICA DE LA FORMACIÓN ARROYO FELICIANO (PLEISTOCENO SUPERIOR) EN LA LOCALIDAD PASO DUARTE, PROVINCIA DE ENTRE RÍOS, ARGENTINA
}

\author{
ALEJANDRO F. ZUCOL ${ }^{1}$ \\ NOELIA I. PATTERER ${ }^{1}$ \\ HERALDO A. LEIVA ${ }^{2}$ \\ MARIANA BREA ${ }^{1}$ \\ ESTEBAN PASSEGGI ${ }^{1}$
}

\begin{abstract}
'Laboratorio de Paleobotánica, Centro de Investigaciones Científicas y Transferencia de Tecnología a la Producción (CICyTTP-CONICET), Dr. Materi y España SN, E3105BWA, Diamante, Entre Ríos, Argentina.

2Pellegrini 798, E3105BWA, Diamante, Entre Ríos.
\end{abstract}

Recibido: 14 de Diciembre de 2015 - Aceptado: 14 de Abril de 2016

Para citar este artículo: Alejandro F. Zucol, Noelia I. Patterer, Heraldo A. Leiva, Mariana Brea y Esteban Passeggi (2016). Caracterización fitolítica de la Formación Arroyo Feliciano (Pleistoceno Superior) en la localidad Paso Duarte, Provincia de Entre Ríos, Argentina. Publicación Electrónica de la Asociación Paleontológica Argentina 16 (1): 52-63.

Link a este artículo: http://dx.doi.org/10.5710/PEAPA.14.04.2016.92

DESPLAZARSE HACIA ABAJO PARA ACCEDER AL ARTíCULO

Asociación Paleontológica Argentina Maipú $6451^{\circ}$ piso, C1006ACG, Buenos Aires República Argentina Tel/Fax (54-11) 4326-7563 Web: www.apaleontologica.org.ar

Otros artículos en Publicación Electrónica de la APA 15(1):

\section{Bardet \& Galoyer}

THE LOST WORLD OF GEORGES CUVIER: MOSASAURIDS FROM

THE CAMPANIAN MEUDON

CHALK (FRANCE).

\section{Bona \& Barrios}

THE ALLIGATOROIDEA OF ARGENTINA: AN UPDATE OF ITS FOSSIL RECORD.

\section{Pereda-Suberbiola et al.}

SIINTESIS DEL REGISTRO FÓSIL DE DINOSAURIOS TIREÓFOROS EN GONDWANA. 


\title{
CARACTERIZACIÓN FITOLÍTICA DE LA FORMACIÓN ARROYO FELICIANO (PLEISTOCENO SUPERIOR) EN LA LOCALIDAD PASO DUARTE, PROVINCIA DE ENTRE RÍOS, ARGENTINA
}

\author{
ALEJANDRO F. ZUCOL ${ }^{1}$, NOELIA I. PATTERER ${ }^{1}$, HERALDO A. LEIVA², MARIANA BREA ${ }^{1}$ Y ESTEBAN PASSEGGI ${ }^{1}$
}

${ }^{1}$ Laboratorio de Paleobotánica, Centro de Investigaciones Científicas y Transferencia de Tecnología a la Producción (CICyTTP-CONICET), Dr. Materi y España SN, E3105BWA, Diamante,Entre Ríos,Argentina.cidzucol@gmail.com; noepatt1@hotmail.com; cidmbrea@gmail.com; estebanpasseggi@cicyttp.org.ar

2Pellegrini 798, E3105BWA, Diamante, Entre Ríos. heraldo_leiva@hotmail.com

Resumen. En esta contribución se da a conocer la asociación fitolítica de una secuencia sedimentaria perteneciente a la Formación Arroyo Feliciano (Pleistoceno Superior), ubicada en la localidad Paso Duarte (provincia de Entre Ríos), alta cuenca del río Gualeguay. El escaso contenido paleontológico hallado hasta la actualidad describe una fauna que se correlacionaría con el Piso/Edad Bonaerense-Lujanense de la provincia de Buenos Aires. Los antecedentes paleobotánicos provienen de los últimos tres años con el registro de leños fósiles que caracterizan ambientes de condiciones climáticas cálidas y húmedas a semi-áridas. El análisis de microrestos en detalle del perfil Paso Duarte, dio como resultado la presencia de diferentes morfotipos fitolíticos cuya asociación zonificó el perfil en tres secciones claramente distinguibles. Los fitolitos observados describen la presencia de una vegetación herbácea dominada por gramíneas meso-megatérmicas de requerimientos climáticos más cálidos y húmedos que los que actualmente prevalecen en el área de la cuenca en estudio.

Palabras clave. Cuaternario. Cuenca del río Gualeguay. Paleoclima. Paleoambiente. Silicofitolitos.

Abstract. PHYTOLITH CHARACTERIZATION FROM THE ARROYO FELICIANO FORMATION (UPPER PLEISTOCENE), PASO DUARTE LOCALITY, ENTRE RÍOS PROVINCE, ARGENTINA. In this contribution a phytolith assemblage of the Arroyo Feliciano Formation (Upper Pleistocene), Paso Duarte locality (Entre Ríos Province), upper Río Gualeguay Basin, was studied. The scarce faunal paleontological record present in the formation have been tentatively correlated with the Bonaerian-Lujanian Stage/Age from Buenos Aires Province, Argentina. The paleobotanical record was studied in the last three years, and is composed by fossil woods that characterize warm and humid to semi-arid environments. The phytolith assemblages of Paso Duarte locality show a composition of elements that allows us to distinguish three sectors along the profile. The analysis of the phytolith assemblages suggests the presence of grasses palaeocommunities, principally dominated by meso-megathermic elements that grew under warm and humid climatic conditions.

Key words. Quaternary. Gualeguay River Basin. Paleoclimate. Paleoenvironment. Silicophytoliths.

GEOMORFOLóGICAMENTE, la región noroeste de la provincia de Entre Ríos se caracteriza por presentar una paleosuperficie correspondiente a la superficie Feliciano-Federal definida por Iriondo y Kröhling (2008), representada por depósitos del Cuaternario que en forma mayoritaria son los que han sido asignados a la Formación Hernandarias (Pleistoceno inferior). Sobre esta paleosuperficie se depositaron unidades fluviales más jóvenes dispuestas en discordancia erosiva por incisión y relleno de valles (Brunetto et al., 2012); tal es el caso de la Formación Arroyo Feliciano, definida en las barrancas del arroyo homónimo en el noroeste de la provincia de Entre Ríos (Iriondo, 1980).

Desde hace unos años la Formación Arroyo Feliciano ha comenzado a estudiarse mediante relevamientos y estudios de materiales fósiles, lo que ha brindado algunos resultados principalmente sobre la paleoflora representada por leños permineralizados (Moya, 2015; Moya y Brea, 2015a,b; Moya et al., 2015).

Como resultado de una primera prospección (Leiva et al., 2013) se realizó un estudio preliminar del contenido fitolítico que forma parte de esta contribución y representa los primeros resultados de este tipo de análisis para la Formación Arroyo Feliciano, en la localidad fosilífera Paso Duarte (Fig. 1, Departamento Federal, provincia de Entre Ríos, Argentina). 


\section{MARCO GEOLÓGICO Y PALEONTOLÓGICO}

El conocimiento de la composición fitolítica de las formaciones geológicas de la provincia de Entre Ríos se ha incrementado en los últimos quince años, abarcando distintos depósitos neógenos de diferentes tipos de ambientes y composición. En la cuenca del río Paraná, Zucol y Brea (2000a,b, 2001) han descripto asociaciones de microfósiles en la Formación Paraná asignada al Mioceno tardío (Tortoniano, 9,47 Ma) (Pérez, 2013) y en la Formación Puerto Alvear (Zucol y Brea, 2005) asignada al Plioceno-Pleistoceno (Iriondo y Kröhling, 2008; Brunetto et al., 2015), mientras que Erra (2010) y Erra et al. $(2011,2013)$ han hecho lo mismo para la Formación Tezanos Pinto del Pleistoceno Superior-Holoceno temprano. En la Cuenca del río Uruguay los estudios han sido realizados principalmente por Patterer $(2012$, 2015) en la Formación San Salvador asignada al Plioceno-Pleistoceno inferior y por Zucol et al. (2005), Patterer (2012), Patterer y Brea (2011) y Patterer et al. (2014) en la Formación El Palmar del Pleistoceno Superior. Los análisis presentados en esta contribución son la primera información sobre la composición fitolítica de sedimentos fluviales pertenecientes a la Cuenca del río Gualeguay, una subcuenca del interior de la provincia de Entre Ríos y afluente de la cuenca inferior del río Paraná, que brinda posibilidades de integrar y/o correlacionar los resultados citados previamente para la provincia de Entre Ríos.

La Formación Arroyo Feliciano (Fig. 1) fue formalmente definida por Iriondo et al. (1985) y representa un relleno aluvial y fluvial acumulado en los valles afluentes de los ríos Paraná y Uruguay en la provincia de Entre Ríos (Argentina), con espesores que varían entre 2 y $6 \mathrm{~m}$. Se compone de depósitos bien seleccionados originados principalmente en ambientes de cauce y de pantano (Iriondo et al., 1985; Iriondo y Kröhling, 2008; Brunetto et al., 2012, 2015). De acuerdo a Iriondo y Kröhling (2008) esta formación fue depositada durante un episodio cálido y húmedo ocurrido durante el Pleistoceno Superior.

La caracterización sedimentológica de la Formación Arroyo Feliciano depende del tamaño y composición geológica de la sub-cuenca fluvial en la que se observe (Iriondo et al., 1985). En detalle y sobre la base del perfil tipo de la formación, ubicado en el arroyo Feliciano en el noroeste de Entre Ríos, $1 \mathrm{~km}$ aguas abajo de la desembocadura del arroyo Las Piedras (Fig. 1), la unidad está compuesta por limos pulverulentos de color gris muy claro a verdoso oliva claro, de consolidación media. Localmente presenta estructuras de laminación y estratificación muy fina; aflora con características masivas sin estratificación visible, con grietas revestidas por óxidos de manganeso. En la sección superior contiene carbonato de calcio segregado en forma de concreciones mal definidas como cemento. Fue interpretada por Iriondo et al. (1985) como facies de Ilanura aluvial depositada bajo un clima húmedo, con introducción posterior del carbonato que ocurrió con un clima semiárido. Las facies de canal de la unidad, en su perfil tipo, están formadas por arenas limosas con estratificación media a gruesa, difusa y con buzamientos singenéticos originados en el relleno de cauces. En la base aparecen depósitos torrenciales formados por cantos rodados carbonáticos o "toscas". Esta unidad constituye la terraza alta de muchos arroyos de la región, caracterizadas por la presencia de paleocauces de gran tamaño. Esto sugiere un cauce estable a lo largo de un tiempo considerable, dicha característica es típica de ríos de climas húmedos sin estación seca (Iriondo y Kröhling, 2008).

Ejemplares de los mamíferos fósiles Toxodon platensis Owen, 1837, Mylodon darwini Owen, 1839, Lestodon sp. (Brunetto et al., 2012) e Hippocamelus sp. (Alcaraz y Ferrero, 2013) han sido registrados en la Formación Arroyo Feliciano y en consecuencia correlacionada con el Piso/Edad Bonaerense-Lujanense de la provincia de Buenos Aires (Brunetto et al., 2012). La gran cantidad y riqueza de taxones de vertebrados incluidos anteriormente en la Formación Arroyo Feliciano y en la Formación Tezanos Pinto (Ferrero, 2009; Brunetto et al., 2015 y referencias citadas allí) es ahora considerada dentro de la Formación Salto Ander Egg. Los análisis estratigráficos, sedimentológicos y cronoestratigráficos fueron realizados por Brunetto et al. (2015), donde se nomina a la Formación Salto Ander Egg como una nueva unidad geológica que forma parte de la secuencia representativa del relleno de valles del Cuaternario superior del sudoeste de la provincia de Entre Ríos. Esta unidad se diferencia claramente de la Formación Arroyo Feliciano por su litología y su contenido paleontológico (Brunetto et al., 2009, 2012; Ferrero, 2013). Ambas formaciones se extienden y afloran en la cuenca de río Gualeguay donde la columna estratigráfica del noroeste está representada por la Forma- 
ción Arroyo Feliciano y la del sudoeste por la Formación Salto Ander Egg (Brunetto et al., 2015). Por otra parte, la presencia de Qualeoxylon felicianense Moya y Brea, 2015 (Vochysiaceae), Cylicodiscuxylon paragabunensis Moya y Brea, 2015 (Fabaceae: Mimosoideae: Mimoseae), Abaremaxylon hydrochorea Moya y Brea, 2015 (Fabaceae: Mimosoideae: Ingaeae) y Styracoxylon thyllosum Moya, Brea y Franco, 2015 (Styracaceae) que son taxones afines a Qualea Aublet, Cylicodiscus Harms, la alliance Abarema Pitter-Hydrochorea Barneby y J.W. Grimens y Styrax L. respectivamente, sugieren que estos ejemplares crecieron bajo condiciones climáticas cálidas y húmedas a semi-áridas (Moya y Brea, 2015 a,b; Moya et al., 2015). Recientemente, se describió también la primera Bignoniaceae afín a las tribus Tecomeae y la alliance
Tabebuia (Moya, 2015).

En la actualidad, la región bajo estudio sobre la cuenca del río Gualeguay se encuentra en la provincia fitogeográfica del Espinal (sensu Cabrera y Willink, 1980), donde la vegetación está dominada por bosques xerófilos bajos y pobres en especies, que se desarrollan bajo un clima cálido y húmedo en el sector norte y seco en el sur, con precipitaciones que varían en valores medios anuales entre 950-1170 mm y una temperatura media anual entre $10-23^{\circ} \mathrm{C}$. Los bosques xerófilos que se encuentran en posiciones del paisaje de media loma y loma están dominados por Acacia caven (Molina) Molina (espinillo), Geoffroea decorticans (Hook. y Arn.) Burkart (chañar), Schinus longuifolia (Lindl.) Speg., (incienso) y Jodina rhombifolia (Hook. y Arn.) Reissek (sombra

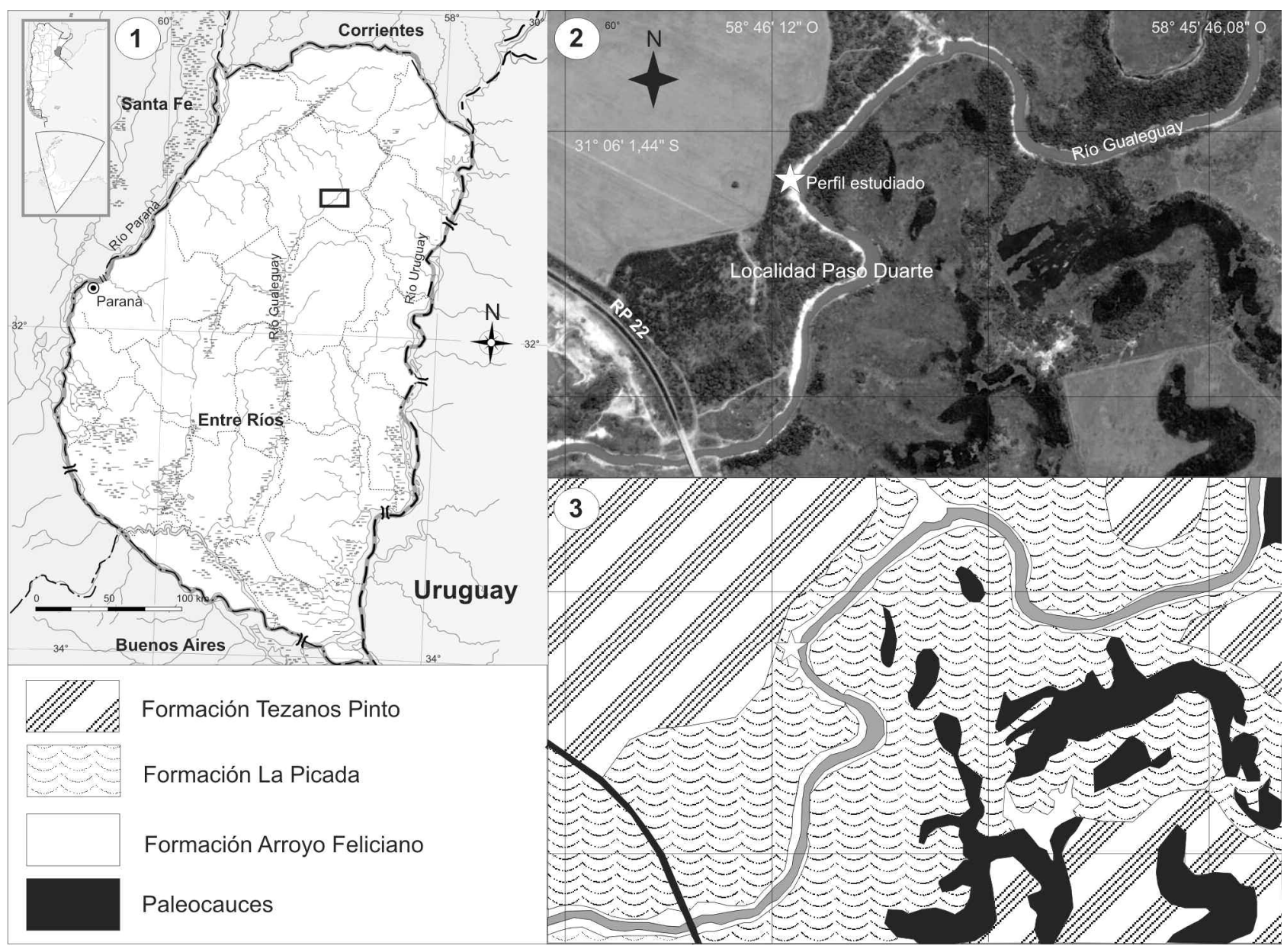

Figura 1. Mapa de la ubicación geográfica del perfil estudiado en la Localidad fosilífera Paso Duarte, Entre Ríos, Argentina. 1, Mapa de la provincia de Entre Ríos con la ubicación del área representada en 2 y 3. 2, Toma de vista satelital. 3, Distribución en superficie de las formaciones geológicas cuaternarias definidas por Iriondo (1980) en la región. 
de toro), mientras que en cauces abandonados y bajos se presentan comunidades de pajonales que en algunos casos llegan a ser comunidades monoespecíficas de Panicum prionitis Ness. (paja de techar). Además, en ambientes aledaños a los cursos de agua es posible encontrar selvas en galería y bosques ribereños empobrecidos en relación a los desarrollados sobre las fajas fluviales directamente vinculados a los ríos Paraná y Uruguay.

\section{MATERIALES Y MÉTODOS}

La localidad Paso Duarte (31 6' S; $58^{\circ} 46^{\prime}$ O) se encuentra en el departamento Federal a orillas del río Gualeguay en la provincia de Entre Ríos (Fig. 1). En ella se muestreó un perfil representativo de la Formación Arroyo Feliciano, teniendo en cuenta su representatividad en el área, su desarrollo y la ausencia de bioturbación o alteración antrópica. Este perfil, denominado Paso Duarte, fue descripto in situ en sus características composicionales (textura y estructura) y, de acuerdo a ello, se realizó una toma de muestras consecutivas definidas por los estratos que lo componían, con una equidistancia de $10 \mathrm{~cm}$ dentro de cada nivel diferenciado. Para evitar la mezcla de muestras se distanciaron las tomas de distintos niveles consecutivos (Fig. 2). Las muestras fueron ingresadas y archivadas en repositorio definitivo bajo la denominación CIDPALBO-MS, del CICyTTP-CONICET de Diamante, Entre Ríos (Zucol et al., 2014).

El perfil Paso Duarte (Fig. 2) posee una potencia superior aflorante de 1,50 m. Es una secuencia granodecreciente caracterizada por estratos limitados por contactos poco visibles, desde la base del mismo y hasta los $50 \mathrm{~cm}$ presenta un estrato principalmente pelítico de color gris con la presencia de arenas gruesas a finas, masivo, con carbonato de calcio y manganeso. Por encima de este, aproximadamente $47 \mathrm{~cm}$, se encuentra un estrato pelítico con una mayor presencia de limos masivos de color pardo grisáceo claro, con óxido de manganeso en grietas y carbonato de calcio, que se continua hacia el techo del perfil por un fino estrato de 3 cm de arcillas masivas pardo grisáceo claro. Los 50 cm suprayacentes presentan textura principalmente arcillosa de color pardo grisácea, muy plástica, con lentes de arcillas oscuras y concreciones de carbonato de calcio. Es importante remarcar que el carbonato de calcio solo se encuentra

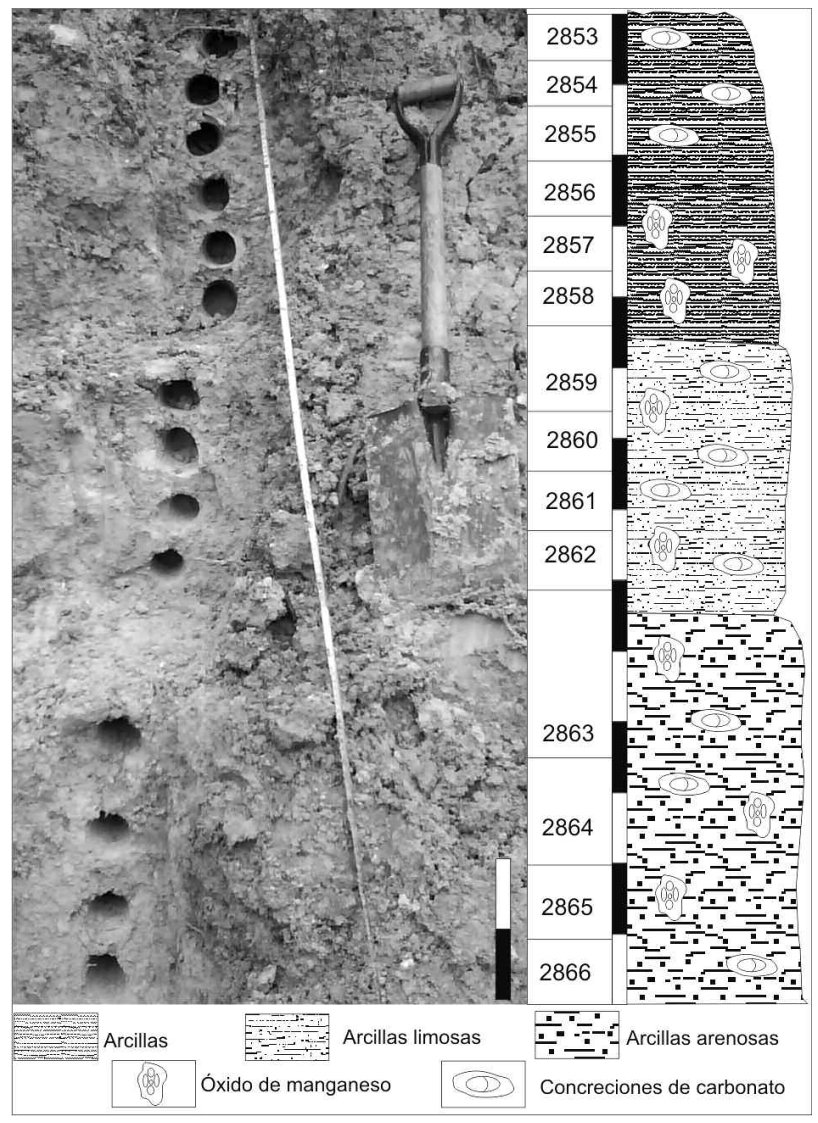

Figura 2. Perfil Paso Duarte, detalle textural-estructural y localización de las muestras sedimentarias. Escala gráfica $=20 \mathrm{~cm}$.

formando concreciones y no en la matriz del depósito. Se diferencian dos secciones, la inferior (muestras -CIDPALBOMS 2866-2859) de naturaleza pelítica-psamítica y la superior (muestras -CIDPALBO-MS 2858-2853), de composición principalmente pelítica, ambas friables.

Para la concentración de fitolitos se siguió el protocolo de procesamiento de Zucol et al. (2010). Para ello se tomaron entre 20-50 gr de muestra y se realizó su limpieza eliminando los distintos compuestos (sales solubles, materia orgánica y carbonatos) que provocan la aglomeración de material silíceo e imposibilitan su correcta observación; posteriormente se realizó una separación granométrica por tamizado para el material grueso y por pipeteado para el material fino, de esta manera se obtuvieron cuatro fracciones: gruesa (>250 $\mu \mathrm{m})$, media $(53-250 \mu \mathrm{m})$, fina $(5-53 \mu \mathrm{m})$ y muy fina $(<5 \mu \mathrm{m})$. Con el material obtenido de las fracciones media y fina se realizó una separación densimétrica me- 
diante el uso de politungstato de sodio como líquido pesado (densidad 2,3). Con el material flotado mediante esta metodología se realizaron los preparados microscópicos utilizando aceite de inmersión y Bálsamo de Canadá como medio de montaje.

Del material proveniente de cada muestra se realizó un recuento de 400 a 500 fitolitos por muestra, usando un microscopio Nikon Eclipse E200. Las fotografías fueron tomadas con una cámara digital Nikon Coolpix S4 anexada al mismo. En la descripción de las asociaciones fitolíticas se hizo hincapié en aquellos morfotipos que se observaron de manera frecuente en las muestras. Dentro de los tipos morfológicos más comunes existe una separación previa en referencia a su tamaño; de esta forma hablamos de macrofitolitos cuando los morfotipos superan los $40 \mu \mathrm{m}$ y microfitolitos cuando son menores a $40 \mu \mathrm{m}$ (sensu Bertoldi de Pomar, 1971, 1975). Los morfotipos fitolíticos no articulados fueron definidos usando la clasificación propuesta por Patterer et al. (2011). Los acrónimos están ejemplificados en la Fig. 1 de la información suplementaria, los que han sido agrupados para su graficación (Tab. 1 de la inf. supl., en donde además se especifica la afinidad botánica de cada uno de estos) según las propuestas de Twiss et al. (1969), Bertoldi de Pomar (1971), Mulholland (1989), Twiss (1992), Kondo et al. (1994), Fredlund y Tieszen (1994) y Zucol (1996) y siguiendo las normativas y descriptores que enumera el International Code for Phytoliths Nomenclature Working Group (ICPNWG, Madella et al., 2005). Los biolitos como espículas de espongiarios, estomatocistes de Chrysostomataceae, diatomeas y otros restos como granos de polen, fueron observados pero no cuantificados. Además de los recuentos realizados en cada muestra (inf. supl., Tabs. 2 y 3 ) se realizó un conteo particular de morfotipos desgastados versus morfotipos de matriz y superficie no alterada (inf. supl. Tab. 4), con el fin de aportar desde la especialidad a una caracterización del agente de transporte y depositación de los materiales que componen la unidad. Con la información de los recuentos se creó una matriz básica de datos, se obtuvo el porcentaje de frecuencia relativa de cada morfotipo fitolítico y se realizó su diagramación mediante el programa Polish Palinological (POLPAL) Numerical Analisis (Walanus y Nalepka, 2000; Nalepka y Walanus, 2003).

\section{RESULTADOS \\ Composición fitolítica}

A modo general se puede observar que las abundancias relativas (inf. supl. Tab. 2 ) de fitolitos en ambas fracciones granométricas $(53-250 \mu \mathrm{m}$ y $53-5 \mu \mathrm{m})$ resultaron equivalentes (Fig. 3), no así el porcentual de elementos desgastados, que se incrementó en ambas fracciones en la sección superior. Entre los macrofitolitos, los originados a partir de elementos buliformes son los que resultaron más abundantes, en particular los de forma poliédrica (Fig. 4, 10-12) y en menor frecuencia los originados a partir de elementos celulares en forma de abanico o flabelos (Fig. 4, 1-6). Los elementos elongados, originados mayormente a partir de células largas epidérmicas, fueron de los más abundantes (Fig. 4, 7-9), mientras que en menor cantidad se encontraron fitolitos originados a partir de células basales de pelos y/o tricomas, fitolitos aciculares o aguzados (Fig. 4, 14-15),

TABLA 1. Valores promedios y rangos del porcentual de fitolitos desgastados en ambas fracciones analizadas, considerados por grupos de asociaciones fitolíticas.

Porcentajes de fitolitos deteriorados

\begin{tabular}{lcc}
\hline & Fracción fina & Fracción media \\
& Mínimo (Promedio) Máximo & Mínimo (Promedio) Máximo \\
Grupo I & $5,43(6,98) 11,34$ & $5,02(8,12) 10,14$ \\
Grupo II & $1,00(4,13) 7,26$ & $7,00(8,68) 10,37$ \\
Grupo III & $9,11(11,50) 16,14$ & $6,88(10,12) 12,71$ \\
Perfil & $1,00(8,19) 16,13$ & $5,02(8,92) 12,71$ \\
\hline \hline
\end{tabular}


los originados en elementos de conducción (Fig. 4, 13) y los elementos de superficie cavada.

Entre los microfitolitos los de forma de conos truncados (Fig. 5, 8-13) y halteriformes (Fig. 5, 1-7) fueron los más abundantes y presentes en toda la sección estudiada, mientras que en menor abundancia y caracterizando solo algún conjunto de asociaciones se presentaron los fitolitos en forma de cruz (Fig. 5, 15 y 16), los halteriformes plurilobados, en forma de silla de montar (Fig. 5, 14), circulares y globulares lisos (Fig. 5, 17-21).

En lo referente a la variabilidad composicional de las asociaciones fitolíticas estimada mediante la rarefacción (Fig. 3) pueden observarse tres principales incrementos 0 picos: uno en la muestra basal CIDPALBO-MS 2866, en la muestra CIDPALBO-MS 2862 de la sección inferior y en la CIDPALBO-MS 2856 de la sección superior, este último de menor cuantía y dado por la variabilidad de los elementos dentro de cada conjunto de morfotipos (Fig. 3). En lo que a su composición respecta, no se presentan grandes variaciones a lo largo de la sección analizada, si bien el análisis multivariado de las asociaciones fitolíticas (CONISS, Constrained Incremental Sum of Squares), permitió establecer tres principales grupos de asociaciones que de piso a techo son: Grupo I (GI), Grupo II (GII) y Grupo III (GIII). El GI corresponde a las muestras de la sección inferior del perfil (ID-CIDPALBO-MS 2066-2060) o los depósitos de composición areno-arcillosa. Estas muestras poseen una asociación fitolítica en donde además de la gran abundancia de fitolitos que fueron descriptos como generales a todo el perfil, se presentan de modo muy frecuente los elongados de bordes lisos (Mp09), los poliédricos de lados rectos, cuadrados, tabulares y tamaño variable (Sx01, Sx02 y Sx03) y los de forma de abanico con flabelo de desarrollo asimétrico (una cara lateral plana y otra cóncava y rematado en forma plana (Fl07, Fig. 4, 4). A su vez es importante la presencia de algunos morfotipos halteriformes, principalmente halterios

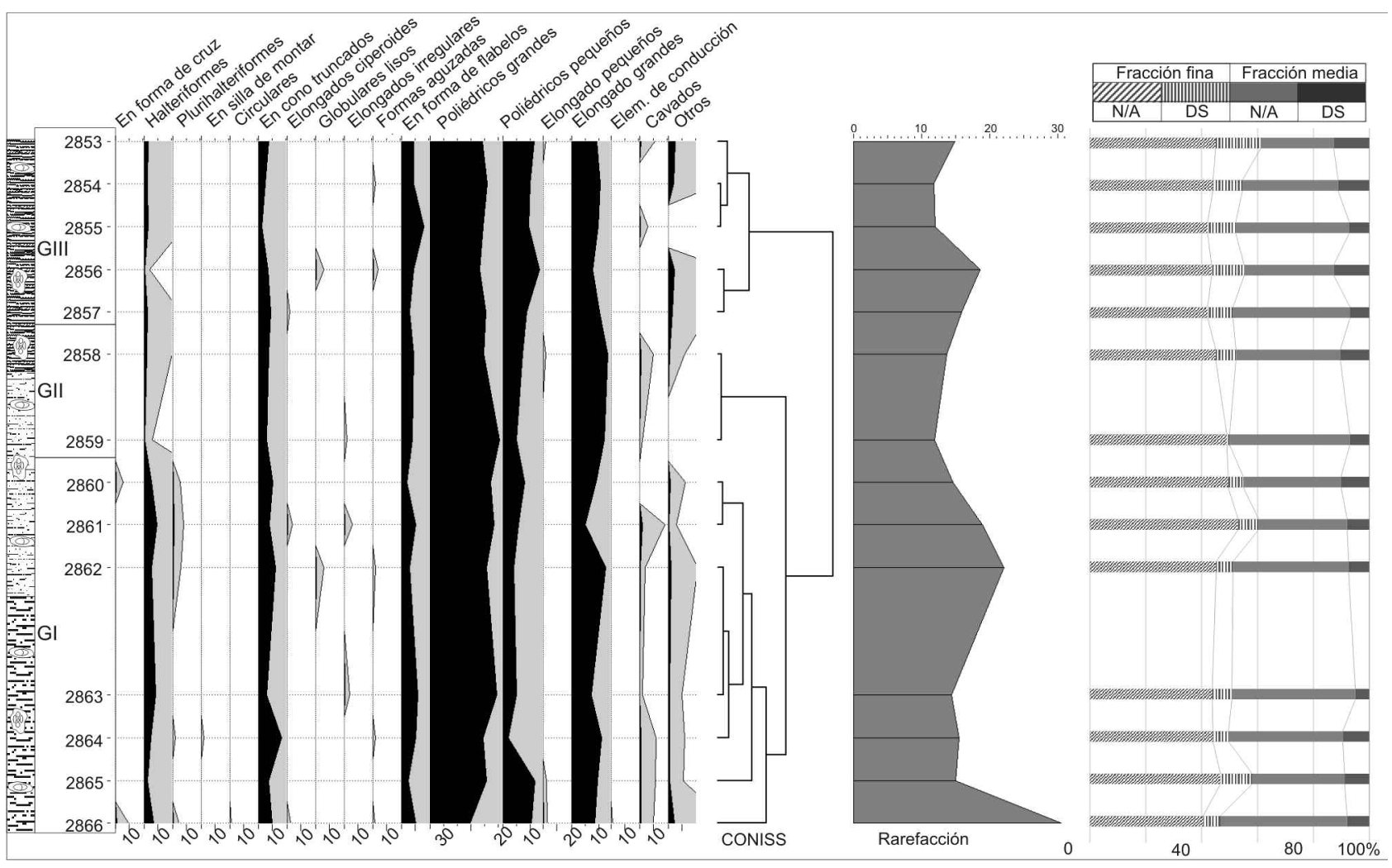

Figura 3. Diagrama fitolítico del perfil Paso Duarte, con el resultado del análisis multivariado (CONISS) y los conjuntos de asociaciones fitolíticas; diagramación de los valores de rarefacción de estas asociaciones y porcentajes de las abundancias de fitolitos de superficies no alteradas y desgastados para las fracciones finas y medianas de cada muestra. Valores en porcentajes en negro y representación en gris con una magnificación x5 de los valores. N/A, no alterados, DS, desgastados. 
con cabezuelas más o menos convexas e istmo poco demarcado (del tipo Ha01, Fig. 5, 3 y 6) y fitolitos en forma de cono truncado achatados, la relación altura/diámetro de estos morfotipos es 1:2 (Ct03, Fig. 5, 9 y 10) y caracterizan a este grupo de asociaciones por su elevada frecuencia.

Los fitolitos esferoidales de superficie lisa solo se presentaron en la muestra 2862. Este grupo de asociaciones se caracteriza también por la presencia diferencial, si bien en menor abundancia o solo en un conjunto de muestras, de elementos halteriformes ( $\mathrm{HaO2}, \mathrm{HaO3}, \mathrm{HaO} 5$ y Ha07), plurilobados con dos istmos y tres lóbulos desarrollados hacia ambos lados (Ph03) y cruces (Ch01, Fig. 5, 15 y 16) con afinidad panicoide y fitolitos en forma de silla de montar de contorno oblado (Sm02, Fig. 5, 14), halteriformes de cabezuelas esféricas (Ha09), ambos de naturaleza chloridoide. Por otra parte, se observaron fitolitos de doble contorno, algunos de ellos de aspecto halteriforme (Ha14) los cuales pueden interpretarse de afinidad estipoide o pooide s.l. Entre los fitolitos elongados fueron frecuentes los largos de contornos lisos (Mp04), de contorno simétrico (Mp06) o aserrados (Mp10), acompañados de flabelos de lados dife-

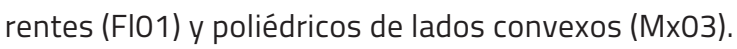

Como elementos que se presentaron en forma exclusiva en la asociación fitolítica de la muestra basal (CIDPALBOMS 2866), debe mencionarse la presencia de fitolitos en forma de cruz y brazos largos (Ch02), halteriformes de afinidad panicoide ( $\mathrm{HaO4}$ ), los de formas de conos truncado y un extremo adelgazado (Ct07) y los de contorno circular a ovados (Ro01) de afinidad estipoide o pooide s.l., también se observaron fitolitos originados en elementos de conducción (Du03), elongados de contorno dendricular (Mp02) y ondulado (Mp08) y formas aguzadas como las Ac02 y Ac04, como parte de la gran variabilidad de esta asociación fitolítica.

En lo que respecta al conjunto de asociaciones fitolíticas, el análisis multivariado (Fig. 3) permite diferenciar a las muestras inferiores (CIDPALBO-MS 2866 y 2865) de las restantes y entre sí, si bien en líneas generales la muestra CIDPALBO-MS 2866 presentó una mayor cantidad y variabilidad de microfitolitos. Las restantes asociaciones se agrupan diferenciándose entre las muestras medias (CIDPALBO-MS 2862-2864) y las dos superiores (CIDPALBOMS 2860 y 2861$)$.
Los porcentajes de fitolitos desgastados (Tab. 1) oscilaron alrededor de una media de 6,98\% para la fracción fina y $8,12 \%$ para la fracción media, en un marco de desgaste promedio de $8,19 \%$ para la fracción fina y $8,92 \%$ para la fracción media del conjunto de las muestras analizadas.

El GIl se conforma por un par de asociaciones (CIDPALBO-MS 2859 y 2858) de composición intermedia entre ambos grupos. Los fitolitos de menor tamaño se encuentran escasamente representados y resultan más abundantes los elementos elongados de bordes lisos (Mp09) y los flabeliformes. Entre estos últimos son muy frecuentes los Fl07 y en menor abundancia los que poseen relación largo ancho del flabelo de 1:1, con flabelo semicircular, convexo y pie de lados convexos (Fl05) y los de pie corto de similar largo que el flabelo, el cual posee una escotadura más o menos pronunciada en su extremo (Fl06), como así también, los poliédricos de largo superior a $30 \mu \mathrm{m}$ (Sx01, Sx02 y $\mathrm{S} \times 03)$.

Dentro de los microfitolitos solo fueron observados fitolitos bilobados de cabezuelas más o menos convexas e istmo poco demarcado ( $\mathrm{HaO1}$ ) y fitolitos en forma de cono truncado (Ct03).

Cabe recordar que entre ambas muestras se incrementó la equidistancia con la finalidad de evitar la zona intermedia entre los depósitos pelíticos-psamíticos inferiores y los pelíticos superiores. No obstante ello, no se ha podido establecer una diferencia en su aspecto composicional entre ambas asociaciones, ni tampoco una caracterización que permita diferenciarlas de las demás asociaciones.

Los porcentajes de desgaste reflejan esta tendencia a una mayor acumulación de fitolitos de tamaño mayor con promedio para la fracción fina de $4,13 \%$ y de $8,69 \%$ para la fracción media.

En la sección superior del perfil, se destaca la presencia del GIII que reúne las asociaciones fitolíticas de las muestras CIDPALBO-MS 2857-2853, estas asociaciones se caracterizan por la presencia de fitolitos elongados (Mp09), poliédricos (Sx01, Sx02, Sx03, Mx01, Mx02 y Mx03) y flabeliformes (Fl02, Fl03, Fl04, Fl05, Fl06 y Fl07) tanto aislados como articulados, que predominaron en todas las muestras del perfil. Los fitolitos bilobados ( $\mathrm{HaO1}$ ) y conos truncados (Ct03) resultaron en muy baja abundancia, mientras que estuvieron ausentes los restantes fitolitos halteriformes y 


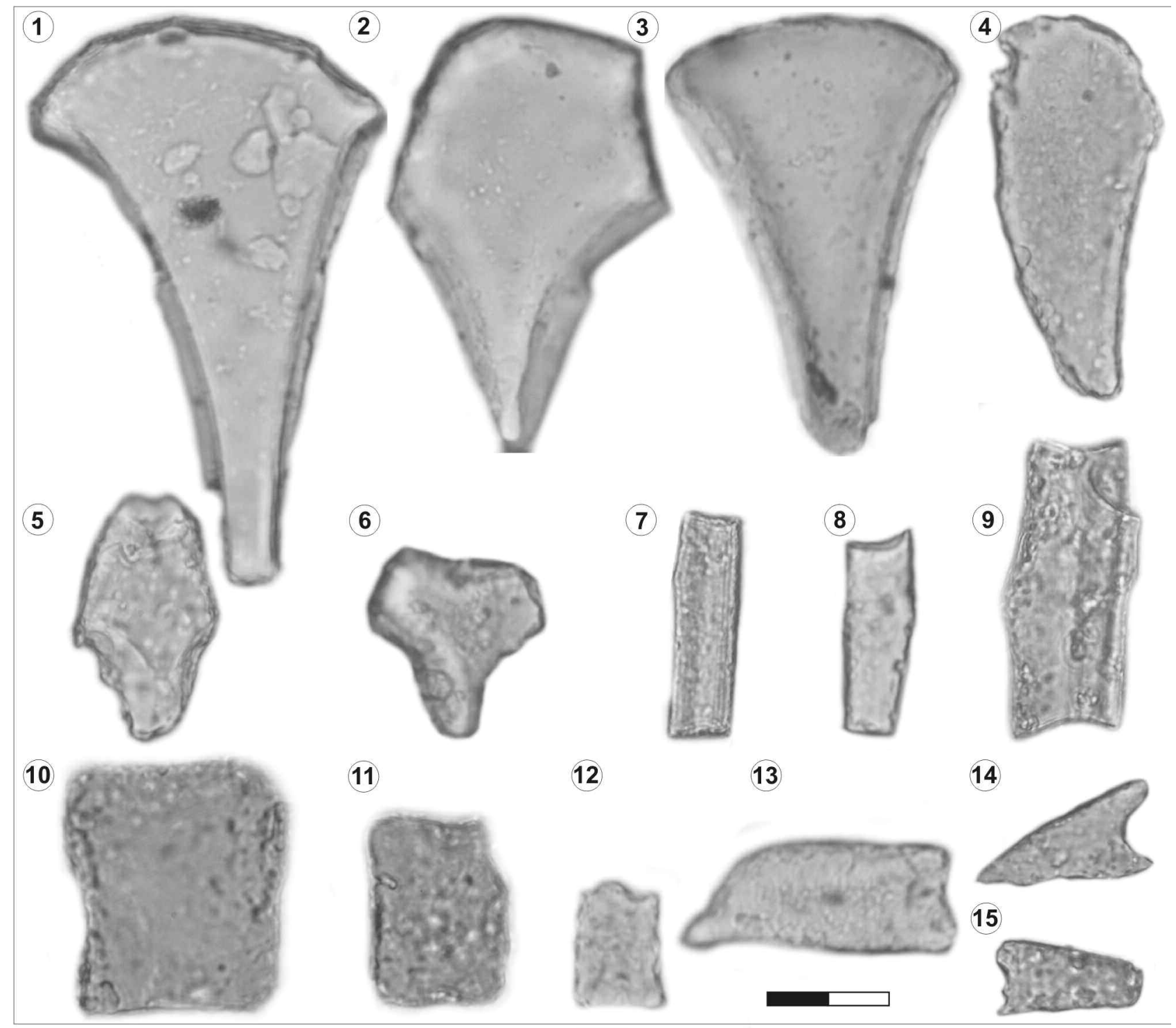

Figura 4. Principales macrofitolitos presentes en el perfil Paso Duarte. 1-6, en forma de flabelo, 1, Fl07, 2 y 5, FlO2, 3, FlO4, 4, FlO3, 6, Fl06; 7-9, elongados, 7 y 8, Mp09, 9, Mp01; 10-12, poliédricos 10, Sx01, 11, Sx03; 12, Mx01; 13, fitolito originado a partir de un elemento de conducción; 14 y 15, aguzados, 14, Ac07, 15, Ac01. Escala gráfica $=40 \mu \mathrm{m}$.

en forma de cruz de afinidad panicoide. Tampoco se observaron los chloridoides, y se reconoció escasa presencia de fitolitos aguzados (Ac01, Ac04 y Ac05) y cavados (Mc01 y Mc03). Los fitolitos esferoidales de superficie lisa solo se presentaron en la muestra 2856. Los porcentajes de desgaste promedio para estas muestras fueron de $11,50 \%$ para la fracción fina y de $10,12 \%$ para la fracción media, lo cual representa un marcado incremento con respecto a los valores presentes para ambas fracciones en los grupos subyacentes.
Las espículas de esponjas estuvieron presentes en la totalidad de las muestras, tanto enteras como fragmentadas; su frecuencia fue mayor en las muestras superiores y se notó una disminución de su presencia a medida que se descendía en el perfil. Los estomastocistes de crisotomatáceas estuvieron presentes, si bien de forma escasa.

\section{DISCUSIÓN}

\section{Interpretación botánica y paleoambiental}

La síntesis presentada en Iriondo y Kröhling (2008) 


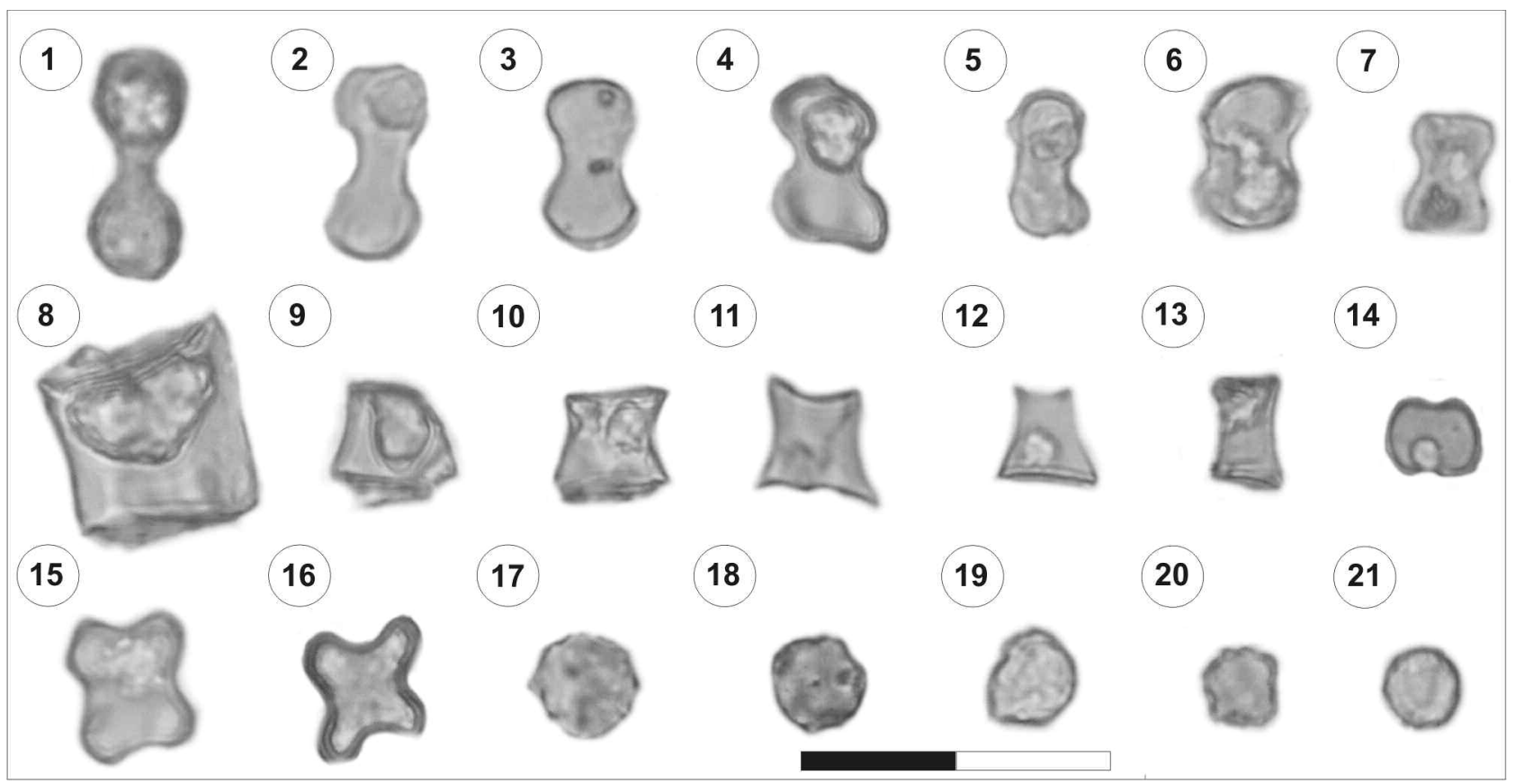

Figura 5. Principales microfitolitos presentes en el perfil Paso Duarte. 1-7, halteriformes, 1, Ha09, 2, HaO4, 3 y 6, HaO1, 4, HaO5, 5, HaO2, 7, Ha08); 8-13, conos truncados, 9 y 10, Ct03, 11 y 12, Ct04, 13, Ct05; 14, en forma de silla de montar Sm01; 15 y 16, en forma de cruces, Ch01; $17-21$, globulares, 17 y 20, GI04, 18, 19 y 21, Gl05. Escala gráfica $=20 \mu \mathrm{m}$.

aporta desde el punto de vista geológico y sedimentológico a la reconstrucción del ambiente depositacional de la Formación Arroyo Feliciano en toda su extensión de distribución. Sin embargo, este trabajo es una de las primeras contribuciones paleobotánicas que aportan al conocimiento del paleoambiente depositacional. Considerando entonces que en el presente trabajo se analiza información puntual de la localidad Paso Duarte, representativo de la alta cuenca del río Gualeguay, resulta importante detallar las características composicionales de las asociaciones de fitolitos haIladas, las cuales muestran variaciones demarcadas entre muestras consecutivas, como así también una zonificación composicional. El conjunto de estos depósitos responde a ambientes fluviales, dado que las características litológicas del perfil estudiado coinciden con las descripciones realizadas por Iriondo et al. (1985) e Iriondo y Kröhling (2008), representando el relleno aluvial y fluvial acumulado en el valle del río Gualeguay.

En este marco, los resultados fitolíticos permiten diferenciar dos principales secciones en el perfil Paso Duarte las cuales presentan fitolitos dominados por elementos gra- miniformes, donde se destaca la presencia de elementos panicoides y danthonioides. Este tipo de elementos estaría caracterizando, para la alta cuenca del río Gualeguay, una vegetación herbácea principalmente de gramíneas meso y megatérmicas, cuyo requerimiento climático es en la actualidad preferentemente cálido y húmedo.

La presencia de restos de espículas de esponjas estaría indicando un ambiente con disponibilidad hídrica y suelos con una alta proporción de humedad o en algunos casos anegados. Existe por otra parte una demarcada diferencia entre el Gl o sección inferior, en donde los elementos megatérmicos (en particular los panicoides y en menor medida los chloridoides) resultan más abundantes y variables con respecto al GIII o sección superior, en donde se observa no solo una disminución de estos elementos sino también una selección granométrica del material depositado. Estas diferencias plantean el interrogante de por qué los depósitos de naturaleza pelítica son los que presentan una menor proporción relativa de microfitolitos. Desde el punto de vista depositacional y considerando los valores de fitolitos desgastados, podrían corresponder a ambientes palustres o al 
menos alejados de los cursos de agua principales, por ejemplo un paleocauce. Trabajos sedimentológicos de detalle son necesarios para reconstruir la dinámica de agradación de los sedimentos.

Además de estos elementos botánicos se hallaron fitolitos de Cyperaceae, Podostemaceae y dicotiledóneas presentes en todo el perfil de modo esporádico, lo cual también responde por estas características a la dinámica del agente fluvial más que a la presencia de esta vegetación a nivel local.

La presencia de fitolitos desgastados y, como en la mayoría de los casos observados, con desgastes superficiales puntuales evidenciados por concavidades (Fig. 4. 4-6, 9-12), indicaría el transporte de elementos a través de corrientes de aguas enérgicas, con valores promedios para ambas fracciones de $8,50 \%$, lo que implica que existió un marcado aporte de la vegetación local. En la comparación por fracción de este tipo de cuerpos desgastados las diferencias de estos porcentuales se mantuvieron entre 0,50-2 en ambas fracciones, con excepción de las muestras medias en donde el porcentaje de fitolitos desgastados de la fracción media resultó superior a los de la fina en un 3-6\%. El análisis de estos porcentajes a lo largo del perfil permite también estimar un marcado incremento de los elementos desgastados desde las muestras inferiores hacia las superiores, por lo cual las interpretaciones paleoflorísticas previas realizadas para el Gl resultarían las que caracterizaron en mayor grado a la vegetación local, si bien son las muestras que menos microfitolitos (elementos en donde se encuentran el mayor número de morfotipos diagnósticos) han presentado.

En el tejido vegetal las células largas presentan un mayor grado de silificación, por lo tanto ofrecen una mayor superficie de ataque con respecto a las células cortas (Madella, 1997; Osterrieth et al., 2009). Los agentes de transporte son un factor importante en la conformación de las asociaciones fitolíticas (Madella y Lancelotti, 2012); el transporte por medio del viento y/o el agua puede provocar la ruptura, astillamiento y/o abrasión de los cuerpos silíceos. En el presente trabajo, los macrofitolitos fueron los que presentaron sus superficies desgastadas con mayor frecuencia, mientras que los microfitolitos pueden evidenciar rupturas además del desgaste superficial mencionado anteriormente. En las asociaciones fitolíticas pueden encon- trarse también fitolitos que no presentan daños físicos (rupturas) notables, lo que se vincula con las fuentes florísticas locales; otros pueden presentar daños que provocan ruptura de los cuerpos como resultado de estrés mecánico consecuencia de un transporte por largas o medianas distancias (Osterrieth et al., 2009). Este tipo de premisas en la interpretación tafonómica de los fitolitos ya ha sido tenido en cuenta y desarrollada en trabajos previos en donde el sedimento muestra un origen netamente fluvial (Zucol et al., 2005; Patterer, 2012). En estos casos se evidencia un marcado aporte de ambientes terrestres aledaños al agente fluvial que permite, de acuerdo a las abundancias, discriminar no solo los elementos que conforman cada grupo de acuerdo al estado de desgaste, sino también la interpretación de carácter local/extralocal de sus integrantes.

\section{Marco paleontológico}

El contenido fósil de naturaleza vegetal de la Formación Arroyo Feliciano incluye Qualeoxylon felicianense, un taxón relacionado con la familia de las Vochysiaceae que en la actualidad se conforma por árboles y arbustos de América tropical (Sytsma et al., 2004; Marcano-Berti, 2005). Este fósil es indicador de condiciones climáticas cálidas y húmedas (Moya y Brea, 2015a). El primer registro fósil para América del Sur de Styracoxylon Burgh, ratifica las condiciones climáticas cálidas y húmedas bajo las cuales se habría desarrollado la formación (Moya et al., 2015). El registro de representantes de la familia Fabaceae con Cylicodiscuxylon paragabunensis y Abaremaxylon hidrochorea corrobora las condiciones climáticas cálidas (Moya y Brea, 2015b), aunque estas autoras mencionan que estos taxones se habrían desarrollado bajo condiciones húmedas a semi-áridas. Esta alternancia de condiciones en la actualidad puede observarse de acuerdo a las condiciones del paisaje en donde coexisten elementos chaqueños con paranaenses, en gradientes altitudinales de escasa distancia.

La Formación Arroyo Feliciano (Iriondo et al., 1985; Iriondo y Kröhling, 2008; Zamboni, 2010) es claramente diferente desde el punto de vista sedimentológico respecto a unidades fluviales cuaternarias como la Formación Salto Ander Egg (Brunetto et al., 2015), ya que esta última unidad posee un mayor contenido relativo de calcita-dolomita (Brunetto et al., 2012). En la actualidad, el registro fósil de la Forma- 
ción Arroyo Feliciano se limita a la presencia de HippocameIus (Artiodactyla: Cervidae), Toxodon platensis (Notoungulata: Toxodontidae), Mylodon darwini y Lestodon sp. (Tardigrada: Mylodontidae). Esta asociación faunística estaría representada por mamíferos cuyos registros son ampliamente conocidos en el Pleistoceno Medio-Tardío, Piso/Edad Bonaerense-Lujanense de la provincia de Buenos Aires.

\section{CONCLUSIONES}

Los antecedentes paleontológicos y paleobotánicos con los que cuenta actualmente la Formación Arroyo Feliciano coinciden en caracterizar paleocomunidades con requerimientos climáticos más cálidos y húmedos que en la actualidad. Estas inferencias paleoclimáticas son coincidentes con las inferidas a partir de los estudios geológicos previos en Entre Ríos (Iriondo y Kröhling, 2008). Los resultados sobre microfósiles silíceos obtenidos en este trabajo, en la sección superior de la unidad en el perfil Paso Duarte, refuerzan esta teoría ya que las asociaciones fitolíticas describen un ambiente de pastizales meso-megatérmicos con disponibilidad hídrica o mayor nivel de humedad en los suelos. Este primer registro fitolítico es importante ya que contribuye a la descripción de la paleoflora para esta secuencia sedimentaria en la alta cuenca del río Gualeguay. Nuevos estudios serán necesarios para corroborar estas primeras inferencias paleofitogeográficas y paleoecológicas de la Formación Arroyo Feliciano.

\section{AGRADECIMIENTOS}

Los autores agradecen a Paulo Pérez Lindo, director del Museo de Antropología y Ciencias Naturales "Conscripto Bernardi" de la ciudad de Federal, Entre Ríos por su colaboración y asistencia durante las tareas de campo. Los autores expresan su agradecimiento a dos revisores anónimos por sus valiosos comentarios y sugerencias que mejoraron el artículo. El presente trabajo fue financiado por la Agencia Nacional de Promoción Científica y Tecnológica (ANPCyT), Proyecto PICT 2008-0176 y Universidad Autónoma de Entre Ríos, PIDP Res. 1423/09 UADER.

\section{BIBLIOGRAFÍA}

Alcaraz, M.A. y Ferrero, B.S. 2013. Hippocamelus Leuckart (Artiodactyla, Cervidae) en el Pleistoceno de la provincia de Entre Ríos, Argentina. Ameghiniana, Suplemento-Resúmenes 50: R5.

Bertoldi de Pomar, H. 1971. Ensayo de clasificación morfológica de los silicofitolitos. Ameghiniana 8: 317-328.

Bertoldi de Pomar, H. 1975. Los silicofitolitos: Sinopsis de su conocimiento. Darwiniana 19: 173-206.

Brunetto, E., Ferrero, B.S., Marino, J., Tentor, F., Sturtz, M., Zamboni, L. y Casanave, L. 2012. Evolución geológica del Pleisto- ceno del centro de Entre Ríos. $5^{\circ}$ Congreso Argentino de Cuaternario y Geomorfología (Río Cuarto), Resúmenes: 1-5.

Brunetto, E., Ferrero, B.S. y Noriega, J.I. 2015. Late Pleistocene lithostratigraphy and sequences in the southwestern Mesopotamia (Argentina): Evidences of the last interglacial stage. Journal South American Earth Sciences 58: 111-128.

Brunetto, E., Ferrero, B.S., Noriega, J.I., Gottardi, M.G., Zamboni, L. y Galina, G. 2009. Formación Salto Ander Egg: depósitos fluviales del Pleistoceno Superior, en el Suroeste de Entre Ríos. $4^{\circ}$ Congreso Argentino de Cuaternario y Geomorfología, $12^{\circ}$ Congresso da Associação Brasileira de Estudos do Quaternário y $2^{\circ}$ Reunión sobre el Cuaternario de América del Sur (La Plata), Actas: p. 260.

Cabrera, A.L. y Willink, A. 1980. Biogeografía de América Latina. Monografía 13 Serie Biología. Secretaria General de la OEA, Washington, p.122.

Erra, G. 2010. Asignación sistemática y paleocomunidades inferidas a partir del estudio fitolítico de sedimentos cuaternarios de Entre Ríos, Argentina. Boletín de la Sociedad Argentina de Botánica 45: 309-319.

Erra, G., Zucol, A.F. y Kröhling, D. 2011. Análisis fitolítico de la Formación Tezanos Pinto (Pleistoceno tardío-Holoceno temprano) en el sector noroeste de su área de distribución en la provincia de Entre Ríos (Argentina). Revista Mexicana de Ciencias Geológicas 28: 398-412.

Erra, G., Zucol, A.F., Kröhling, D.M. y González, C.A. 2013. Análisis Fitolítico de la Formación Tezanos Pinto (Pleistoceno tardíoHoloceno temprano) en el sector sudoeste de la provincia de Entre Ríos (Argentina). Ameghiniana 50: 429-446.

Ferrero, B.S. 2009. [Diversidad y evolución de los Mamíferos del Cuaternario de la provincia de Entre Ríos, Argentina. Aspectos bioestratigráficos y paleozoogeográficos de una fauna particular. Tesis Doctoral, Universidad Nacional de la Plata, Buenos Aires, 425p. Inédita.].

Ferrero, B.S. 2013. Los vertebrados del Cuaternario de la provincia de Entre Ríos. Ameghiniana, Suplemento-Resúmenes 50: R11.

Fredlund, G.G. y Tieszen, L.T. 1994. Modern phytolith assemblages from the North American Great Plains. Journal Biogeography 21: 321-335.

Iriondo, M.H. 1980. El cuaternario de Entre Ríos. Revista de la Asociación de Ciencias Naturales del Litoral 11: 125-141.

Iriondo, M.H. y Kröhling, D. 2008. Cambios ambientales en la cuenca del Uruguay (desde el Presente hasta dos millones de años atrás). Colección Ciencia y Técnica, Universidad Nacional del Litoral, Santa Fe, p. 330.

Iriondo, M., Tardivo, R.N. y Ceruti, C.N. 1985. Geomorfología y cuaternario del tramo inferior del arroyo Feliciano (Entre Ríos, Argentina). Revista de la Asociación de Ciencias Naturales del Litoral 16: 149-156.

Kondo, R., Child, C. y Atkinson, I. 1994. Opal phytoliths of New Zealand. Maanaki Whenua Press, Lincoln, p. 85.

Leiva, H., Patterer, N.I., Zucol, A.F. y Brea, M. 2013. Asociación Fitolítica del perfil Paso Duarte de la Formación Arroyo Feliciano (Pleistoceno Superior), Entre Ríos, Argentina. Ameghiniana, Suplemento-Resúmenes 50: R14.

Madella, M. 1997. Phytolith analysis from the Indus Valley site of Kot Diji, Sindh, Pakistan. En: A. Sinclair, E. Slater y J. Gowlett (Eds.), Archaeological Sciences. Oxbow, Oxford, p. 294-302.

Madella, M., Allexandre, A. y Ball, T. 2005. International Code for Phytolith Nomenclature 1.0. Annals of Botany 96: 253-260.

Madella, M. y Lancelotti, C. 2012. Taphonomy and phytoliths: A user manual. Quaternary International 275: 76-83.

Marcano-Berti, L. 2005. Vochysiaceae. En: P.E. Berry, K. Yatskievych 
y B.K. Holst (Eds.), Flora of the Venezuelan Guayana. RutaceaeZygophyllaceae. Missouri Botanical Garden Press, Saint Louis, p. $500-524$.

Moya, E. 2015. Bignoniaceae fósil en la Formación Arroyo Feliciano (Pleistoceno Superior), Entre Ríos, Argentina. Boletín de la Sociedad Argentina de Botánica, Suplemento 50: 61.

Moya, E. y Brea, M. 2015a. A new record of fossil wood of Vochysiaceae from the late Pleistocene (Arroyo Feliciano Formation), Argentina, South America. Revista Brasilera de Paleontologia 18: 83-90.

Moya, E. y Brea, M. 2015b. Legume fossil woods from the Arroyo Feliciano Formation (late Pleistocene, Northeastern Argentina): paleobiogeographic implications. Ameghiniana 52: 558-573.

Moya, E., Brea, M. y Franco, J. 2015. First record of Styracoxylon (Styracaceae) from Southern Hemisphere: Arroyo Feliciano Formation (Upper Pleistocene), Entre Ríos, Argentina. Review Paleobotany and Palynology 221: 211-229.

Mulholland, S.C. 1989. Phytolith shape frequencies in North Dakota grasses: a comparison to general patterns. Journal Archaeology Science 16: 489-511.

Nalepka, D. y Walanus, A. 2003. Data processing in pollen analysis. Acta Paleobotánica 43: 125-134.

Osterrieth, M., Madella, M., Zurro, D. y Álvarez, M.F. 2009. Taphonomical aspects of silica phytoliths in the loess sediments of the Argentinean Pampas. Quaternary International 193: 70-79.

Patterer, N.I. 2012. [Análisis Fitolíticos de la Formación El Palmar (Pleistoceno superior) en la cuenca del río Uruguay (este de Argentina). Tesis Doctoral, Universidad Nacional de Córdoba, Córdoba, 307 p. Inédita.].

Patterer, N.I. 2015. Análisis fitolíticos de la Formación San Salvador (Plioceno-Pleistoceno inferior) en la Cuenca del río Uruguay, Entre Ríos, Argentina. Revista de la Asociación Geológica Argentina 72: 332-344.

Patterer, N.I. y Brea, M. 2011. Phytolith Analysis in fluvial sediments of El Palmar Formation (late Pleistocene) in eastern Argentina. 8th International Meeting on Phytolith Research (Colorado), Abstracts: 42-43.

Patterer, N.I., Passeggi, E. y Zucol, A.F. 2011. Análisis fitolíticos de suelos del sudoeste de la Provincia de Entre Ríos (Argentina) como una herramienta para comprender sus procesos pedológicos. Revista Mexicana de Ciencias Geológicas 28: 132-146.

Patterer, N.I., Zucol, A.F. y Brea, M. 2014. Análisis fitolíticos del perfil sedimentario Los Loros, de la Formación El Palmar (Pleistoceno superior), en el Parque Nacional El Palmar, Entre Ríos, Argentina. Acta Geológica Lilloana 26: 53-62.

Pérez, L. 2013. Nuevos aportes al conocimiento de la edad de la Formación Paraná, Mioceno de la provincia de Entre Ríos, Argentina. En: D. Brandoni y J.I. Noriega, (Eds.), El Neógeno de la Mesopotamia argentina. Publicación Especial de la Asociación Paleontológica Argentina, 14: 7-12.

Sytsma, K.J., Litt, A., Zjhra, M.L., Pires, J.C., Nepokroeff, M., Conti, E., Walker, J. y Wilson, P.G. 2004. Clades, clocks, and continents: historical and biogeographical analysis of Myrtaceae, Vochysiaceae, and relatives in the southern hemisphere. International Journal of Plant Sciences 165: S85-S105.

Twiss, P.C. 1992. Predicted world distribution of C3 and C4 grass phytoliths. En: G. Rapp y S.C. Mulholland (Eds.), Phytoliths Systematics, Emerging Issues. Springer, Nueva York, p. 113-128.

Twiss, P.C., Suess, E. y Smith, R.M. 1969. Morphological classification of grass phytoliths. Soil Science Society of America Proceedings 33: 109-115.

Walanus, A. y Nalepka, D. 2000. POLPAL, Counting pollen, tables storage and diagrams plotting. System manual. Polish Academy of Sciences, W. Szafer Institute of Botany, Cracovia, p. 25.

Zamboni, L.D. 2010. [Comparación geológica y de contenido fosilífero de las terrazas fluviales de dos localidades de la provincia de Entre Ríos. Tesina, Facultad de Ciencia y Tecnología, Universidad Autónoma de Entre Ríos, Entre Ríos. p. 45. Inédita.].

Zucol, A.F. 1996. Microfitolitos de las Poaceae argentinas: I. Microfitolitos foliares de algunas especies del género Stipa (Stipeae: Arundinoideae), de la Provincia de Entre Ríos. Darwiniana 34: $151-172$.

Zucol, A.F. y Brea, M. 2000a. Análisis fitolítico de la Formación Paraná (Mioceno superior) en el Departamento Diamante, Entre Ríos, Argentina. $2^{\circ}$ Congreso Sedimentológico Latinoamericano y $8^{\circ}$ Reunión Argentina de Sedimentología (Mar del Plata), Resúmenes: 190-191

Zucol, A.F. y Brea, M. 2000b. Análisis fitolítico de la Formación Paraná en Entre Ríos. En: F. Aceñolaza y R. Herbst (Eds.), El Neógeno de Argentina. INSUGEO, Serie de Correlación Geológica, 14: 67-76.

Zucol, A.F. y Brea, M. 2001. Asociación fitolítica de la Formación Alvear (Pleistoceno Inferior), Entre Ríos, Argentina. Ameghiniana, Suplemento-Resúmenes 38: R49.

Zucol, A.F. y Brea, M. 2005. Fitolitos: IV. Sistemática de fitolitos, pautas para un sistema clasificatorio. Un caso en estudio en la Formación Alvear (Pleistoceno inferior). Ameghiniana 42: 685-704.

Zucol, A.F., Brea, M., Passeggi, E. y Franco, M.J. 2014. Colecciones del laboratorio de paleobotánica y procesamiento de material sedimentario del CICYTTP- DIAMANTE (CONICET), Entre Ríos, Argentina. Boletín de la Asociación Latinoamericana de Paleobotánica y Palinología 14: 71-82.

Zucol, A.F., Brea, M. y Scopel, A. 2005. First record of fossil Wood and phytoliths assemblages of the Upper Pleistocene in El Palmar National Park (Argentina). Journal of South American Earth Sciences 20: 33-43.

Zucol, A.F., Passeggi, E., Brea, M., Patterer, N.I., Fernández Pepi, M.G. y Colobig, M.M. 2010. Phytolith analysis for the Potrok Aike lake drilling proyect: Protocols of sample treatment in the frame of the PASADO microfossil manual. En: H. Corbella y N.I. Maidana (Eds.). $1^{\circ}$ Reunión Internodos del Proyecto Interdisciplinario Patagonia Austral y $1^{\circ}$ Workshop Argentino del Proyecto Potrok Aike Maar Lake Sediment Archive Drilling Project, Proyecto Editorial PIPA (Buenos Aires), p. 81-84.

Doi: 10.5710/PEAPA.14.04.2016.92

Recibido: 14 de Diciembre de 2015

Aceptado: 14 de Abril de 2016 